\title{
Measurement of aqueous cells and flare in normal eyes
}

\author{
S M Shah, D J Spalton, S E Smith
}

\begin{abstract}
The Kowa laser cell flare meter has been recently introduced to quantify the assessment of aqueous cells and flare in vivo by measurement of light scattering from a low power He$\mathrm{Ne}$ beam. Computer analysis of this scattered light can distinguish cells (expressed as cell count) from protein (expressed as photon count/ms), and in-vitro work with albumin solutions has shown that the photon count/ms is linearly related to protein concentration. This study of 106 normal eyes (53 subjects) assesses the accuracy and sensitivity of the instrument and the factors affecting the interpretation of its results. Using in-vitro solutions of human albumin we found a highly significant linear correlation $(r=1, p=0.0001)$ between photon count and protein concentration. The results show that the instrument has a photon count/ms reproducibility of $8 \cdot 26 \%$ and that there is a within-subject variability in photon count/ms (aqueous flare) of $12 \cdot 2 \%$ in normal eyes. No significant difference in photon count/ms (aqueous flare) was found to exist between right and left eyes ( $p>0.4)$, between sexes $(p>0.5)$, or between irides of different colour $(p>0.8)$. There was also no statistically significant variation in photon counts/ms with time of day over the period of measurement $(1000-1900 \mathrm{~h})(\mathrm{p}=0 \cdot 4)$. There was, however, an increase in photon count $/ \mathrm{ms}$ with age $(r=0.57$, $p<0.001$ ) and a decrease with pupillary dilatation at both 30 and 60 minutes after instillation of tropicamide $1 \%(p<0 \cdot 05)$. An occasional cell was found in only $10.4 \%$ of normal eyes, and there was no significant increase in the cell counts on mydriasis $(p>0.05)$. These findings indicate that the Kowa laser flare meter is an accurate and sensitive instrument with potential application in the investigation of the blood-aqueous barrier.
\end{abstract}

Breakdown of the blood aqueous barrier is manifested clinically as flare and cells in the anterior chamber of the eye. Assessment of these parameters is of fundamental clinical importance in anterior segment surgery, inflammatory ocular disease, and potentially in surgical audit. Up to now it has not been possible to quantify this response in a simple non-invasive manner.

The Kowa FC1000 laser flare cell meter is a recently introduced instrument that allows a quantitative measurement of flare and cells by a non-invasive technique which depends on scanning the anterior chamber with a $\mathrm{He}-\mathrm{Ne}$ laser and measurement and analysis of the scattered light. The interpretation of clinical data with this machine requires a knowledge of the variations found in normal eyes. We report a study of 53 normal subjects (106 eyes).

\section{Materials and methods}

\section{INSTRUMENT}

The Kowa laser flare cell meter consists of a He-Ne laser slit-lamp, a binocular microscope fitted with a photomultiplier, and a personal computer. The He-Ne beam has a power of 25 $\mu \mathrm{W}$ and a diameter of $20 \mu \mathrm{m}$. The beam is projected into the anterior chamber, and scattering of the beam within a sampling window $(0.3 \times 0.5 \mathrm{~mm})$ is detected by the photomultiplier. Two measurement modes are used, one for protein concentration and the other for cell count. Computer analysis can distinguish between photons reflected from cells and protein, and in-vitro work has shown that the photon count/ms with albumin solutions is directly proportional to the concentration in solution. ${ }^{1}$

Protein concentration measurement mode. The laser beam is scanned vertically for a length of 0.6 $\mathrm{mm}$, covering the sampling window. Scattering of this beam is measured when it passes through this window. Measurements are also taken when the beam passes above and below the sampling window to assess the background signal (BG1 and BG2). The flare value in photon counts/ms is calculated by subtracting the mean of the two background counts from the signal value.

Cell count mode. The beam is scanned two dimensionally, $0.6 \times 0.25 \mathrm{~mm}$ in the sampling window and the scanning volume corresponds to $0.075 \mathrm{~mm}^{3}$. A strong peak of scattered light is obtained when the beam strikes a floating cell, and the number of peaks is counted by the computer.

The instrument automatically converts from one mode to the other. Each mode takes 0.5 second, and thus the total measurement time is 1 second. Scans in which the difference between BG1 and BG2 exceeds $15 \%$, indicative of a nonuniform background, are excluded, and the scan is repeated. Once the eye was aligned the anterior chamber was scanned five times. Each sequential scan was accepted provided the inconsistency between background counts was less than $15 \%$.

\section{CALIBRATION}

To calibrate the instrument freshly prepared solutions of pure human albumin in saline $(0.9 \%$ $\mathrm{NaCl})$ over the concentration range $0-10000$ $\mathrm{mg} / \mathrm{l}$ were placed in plastic cuvettes. These cuvettes were then scanned and an average of five consecutive measurements taken. This procedure was repeated twice at each protein concen- 
tration. A cuvette filled with $0.9 \%$ saline but with no albumin was used as a control.

\section{SUBJECTS}

Strict exclusion criteria were applied to select subjects to ensure normality. Subjects with diabetes, hypertension, renal and hepatic disease, or other significant systemic diseases were excluded, as were subjects with any past or present ocular disease. The use of any regular systemic or topical ocular medication or the recent use of anti-inflammatory drugs also excluded subjects. All subjects satisfying these criteria were then examined ophthalmologically, and only those with a visual acuity of $6 / 6$ or better with no clinical evidence of anterior chamber activity or lens opacity were chosen for final inclusion in the study. Iris colour was classified as blue, Caucasian brown, Asian brown, and Negro brown.

The informed consent of each subject was obtained after the nature of the procedure had been explained fully.

\section{FLARE VALUES}

Reproducibility. Flare values were determined on both eyes of 17 normal subjects, and these measurements were repeated after an interval of five minutes. The variation between the first and second measurements was derived from the within-subject, within-eye, residual mean square and expressed as a coefficient of variation.

Subject variability. Flare values were determined in both eyes of 17 normal subjects, and these measurements were repeated one week later at the same time of day. The variation between the first and second measurements was then assessed in the same manner and expressed as a coefficient of variation.

Factor analysis. Flare values and cell counts were determined in 53 normal subjects (106 eyes) between 1000 and $1900 \mathrm{~h}$. Differences between eyes and individual subjects were then analysed by multiple regression analysis to assess the effect of eye $(R / L)$, sex, iris colour, time of day, and age on photon counts/ms.

Pupillary dilatation. Flare values and cell counts were determined in 10 normal subjects. The left eye of each subject was then dilated with tropicamide $1 \%$, and the measurements were repeated at 30 minutes and 60 minutes after instillation of drops.

Figure 1 Photon count and protein concentration. Scatter plot of the log of protein concentration (human albumin (mg/dl)) against the log of photon count (/ms) with a superimposed line of linear regression $(y=1 \cdot 35 x+0 \cdot 2$, $r=1, p=0 \cdot 0001) .(S I: m g /$ $d l=m g \times 10 / l$.)

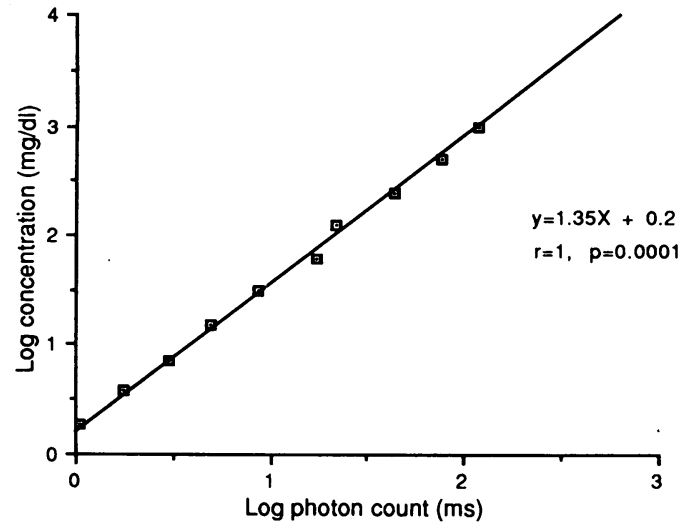

Results

CALIBRATION

A highly significant linear correlation $(r=1$, $\mathrm{p}=0.0001$ ) was found to exist between photon count $(/ \mathrm{ms})$ and the protein concentration in solution over the range $0-1000 \mathrm{mg} / \mathrm{dl}(\mathrm{S} 1: 0$ $10000 \mathrm{mg} / \mathrm{l}$ ) (Fig 1). Using the linear regression formula obtained $(y=1.35 x+0.2$ where $y=\log$ of protein concentration $(\mathrm{mg} / \mathrm{l})$ and $\mathrm{x}=\log$ of photon count $(/ \mathrm{ms})$ ) it is possible to convert photon count into a protein concentration expressed in $\mathrm{mg} / \mathrm{l}$ of human albumin.

\section{REPRODUCIBILITY}

There was no significant difference between the photon counts/ms (mean and SD) due to flare on the first $(4.43,1.09)$ and second $(4.28,1.08)$ measurements and the average coefficient of variation between the two measurements, expressed as a percentage was $8 \cdot 26 \%$. With the formula:

$$
\text { Coefficient of reproducibility }=\frac{(|x-y|)}{(x+y) / 2}
$$

as used by Oshika et $a l{ }^{2}$ where $\mathrm{x}$ is the first measurement and $y$ the second, the value becomes $9 \cdot 54 \%$.

\section{SUBJECT VARIABILITY}

There was no significant difference between the first $(3.98,0.81)$ and second $(3.91,0.81)$ measurements (mean, SD) taken one week later at the same time of day, and the average coefficient of variation between the two measurements, expressed as a percentage, was $12 \cdot 2 \%$ or $13.3 \%$ by the formula adopted by Oshika et al. ${ }^{2}$

\section{MULTIPLE REGRESSION ANALYSIS}

The average age of the 53 normal subjects was 37.9 (SD 15.7) years range 17-81 years. There were 24 male and 29 female subjects. The average photon count/ms for the entire group $(n=106)$ was $4 \cdot 24(\mathrm{SD} 1 \cdot 12)$, range $1 \cdot 8-8 \cdot 2$, and the overall average measurement variation was $25 \cdot 1 \%$ (SD $13 \cdot 1 \%$ ). With our calibration curve and the linear regression formula this mean photon count (per ms) of $4 \cdot 24$ corresponds to a protein concentration of $111 \mathrm{mg} / \mathrm{l}$ of human albumin. The average cell count for the entire group was 0.02 (SD 0.06 ), range $0-0.2$, cells not being detected in $89.6 \%$ of eyes. The remaining $10.4 \%$ registered only 1 cell on one of the five scans, giving a cell count of 0.2 per person.

Multiple regression analysis revealed that there were no significant differences between eyes $(p>0.4)$, between sexes $(p>0.5)$, or between subjects with different coloured irides $(\mathrm{p}>0.8)$ (Table 1). There was also no statistically significant variation in photon count/ms with time of day over the period of measurement $(1000-1900)(p=0 \cdot 4)$. There was a positive correlation between age and photon counts/ms (correlation coefficient $\mathrm{r}=0.57, \mathrm{p}<0.001$ ) (Table 2, Fig 2). A one-way analysis of variance showed that there was a variation in photon counts/ms grouped by age as in Table 2 $(p=0.0001)$. Unpaired $t$ tests then showed that 
Table 1 Iris colour and photon count/ms

\begin{tabular}{lll}
\hline Iris & No. of eyes & $\begin{array}{l}\text { Photon count/ms, } \\
\text { mean }(S D)\end{array}$ \\
\hline Blue & 48 & $4 \cdot 19(1 \cdot 32)$ \\
All brown & 58 & $4 \cdot 12(1 \cdot 05)$ \\
Caucasian brown & 28 & $4 \cdot 15(1 \cdot 25)$ \\
Asian brown & 14 & $3 \cdot 83(0 \cdot 57)$ \\
Negro brown & 16 & $4 \cdot 32(0 \cdot 98)$ \\
\hline
\end{tabular}

Table 2 Age and photon count/ms

\begin{tabular}{llll}
\hline $\begin{array}{l}\text { No of } \\
\text { subjects }\end{array}$ & Age & $\begin{array}{l}\text { Photon count/ms, } \\
\text { mean }(S D)\end{array}$ & $p$ \\
\hline 24 & $<30$ & $3.68(0.59)$ & - \\
9 & $30-40$ & $3.94(0.60)$ & $0 \cdot 27$ \\
8 & $41-50$ & $4.47(0.72)$ & $0.004^{\star}$ \\
5 & $51-60$ & $5.15(1.65)$ & $0.0014^{\star}$ \\
5 & $61-70$ & $5.25(1.06)$ & $0 \cdot 0001^{\star}$ \\
2 & $>70$ & $6.52(1.24)$ & $0.0001^{\star}$
\end{tabular}

The $\mathrm{p}$ value was calculated by the unpaired two-tailed $t$ test comparing mean photon count $/ \mathrm{ms}$ of each age group with the mean value of the $<30$ age group

$\star$ Statistically significant $(\mathrm{p}<0.05)$.

the photon counts/ms were significantly higher in all age groups when compared with photon counts $/ \mathrm{ms}$ in the $<30$ age group, and the level of the statistical significance was found to increase with age.

For future research studies it is important to know how small a difference could be detected between the parameters in which no difference was found. The following equation can be used to determine this:

$$
\mathrm{n}=2\left(\frac{\boldsymbol{\sigma}}{\delta}\right)^{2}(\mathrm{Z} \alpha / 2+\mathrm{Z} \beta)^{2}
$$

where $\mathrm{n}=$ the number of subjects, $\sigma$ is the standard deviation, $\delta$ is the smallest detectable difference, $\alpha$ is the significance level and $1-\beta$ is the statistical power and $Z \alpha / 2$ and $Z \beta$ are the ordinates for the normal distribution. ${ }^{3}$ The factor of 2 is not required in calculations using paired data for right and left eyes.

This equation assumes that the photon counts/ ms measured with this instrument in the different groups are approximately normally distributed with a range of standard deviations similar to that found in this study.

With the pooled standard deviation values in the case of unpaired data obtained in our study of 53 subjects and setting the significance level at $5 \%$, and the statistical power at $90 \%$, the smallest difference that could have been detected was 0.48 photon $/ \mathrm{ms}$ for the study of right and left eyes (with $n=53, \sigma=1.069$ ), 1.03 photons/ms for the study on the effect of sex (with $n=24$, $\sigma=1 \cdot 103$ ), and 1.02 photons $/ \mathrm{ms}$ for the study on the effect of iris colour (with $n=24, \sigma=1 \cdot 094$ ).

Figure 2 Photon count/ms and age. Scatter plot of photon count/ms $(n=106)$ versus age, with a superimposed line plot of the calculated mean, and upper and lower $95 \%$ confidence limits.

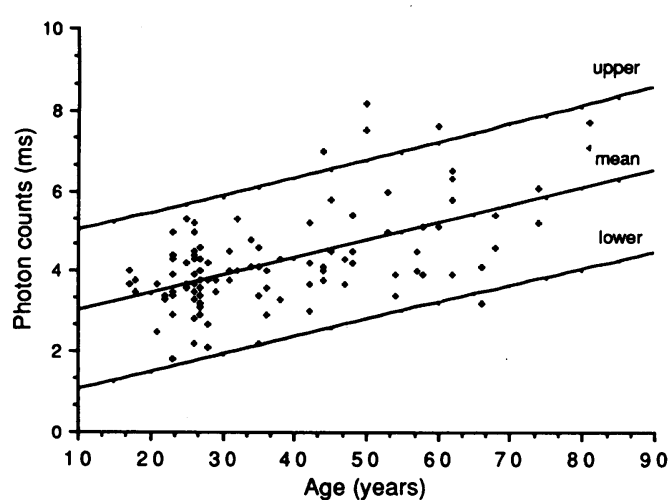

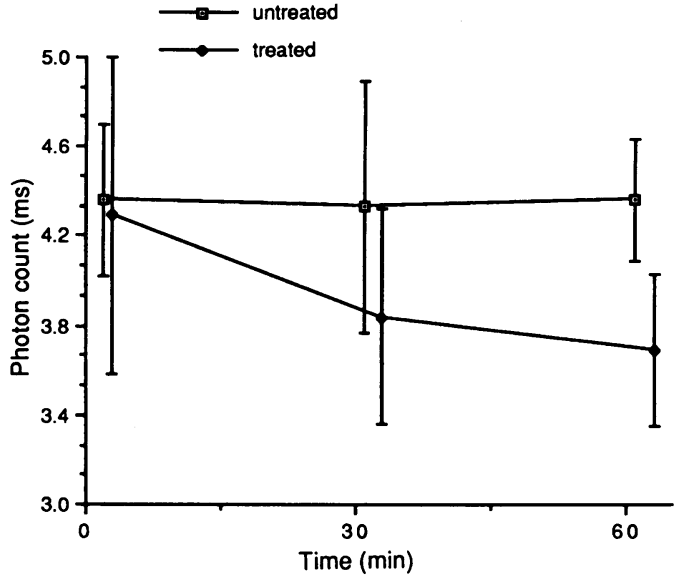

Figure 3 Photon count/ms and pupillary dilatation. Mean photon counts/ms (bars =1 SD) in both the treated and untreated eyes of 10 normal subjects, prior to and at 30 and 60 minutes after instillation of tropicamide $1 \%$ into the treated eye. The decrease in photon counts/ms in the treated eye is statistically significant at both 30 minutes ( $p=0.03$ ) and 60 minutes $(p=0.01)$ after instillation of tropicamide

$1 \%$. The ratio of photon counts/ms between the untreated and treated eye at 0 minutes was 0.98 (SD 0.12) and at 60 minutes was 0.85 (SD 0.09), this decrease being statistically significant $(p=0.0005)$.

\section{PUPILLARY DILATATION}

The results are presented in Figure 3 and Table 3. A one-way analysis of variance of photon counts/ms in the treated eye grouped by time of measurement $(0,30,60$ minutes) showed a significant variation between the groups $(p=0.04)$. A similar test on the untreated eye showed no such difference $(p=0.98)$. The data were then further analysed by a paired two-tailed $t$ test. There was no significant difference between photon counts/ms for the right and left eyes prior to dilatation. Photon counts/ms decreased significantly in the eye with the dilated pupil at 30 minutes $(p=0.03)$ and 60 minutes $(p=0.01)$, with no significant change in photon counts/ms in the untreated eye. The difference between the photon counts/ms in right and left eye at 30 and 60 minutes was also statistically significant $(p=0.01$ and $p=0.0005$ respectively).

The mean (with SD) photon count/ms for the treated eye at 60 minutes was $3.65(0.34)$ and for the untreated eye was $4 \cdot 32(0 \cdot 27)$. The ratio of counts in the treated/untreated eye was 0.847 $(0.097)$ compared with $0.982(0.12)$ prior to dilatation. This change in ratio was highly significant $(p=0.002)$.

In the untreated eye the mean background count, $(\mathrm{BG} 1+\mathrm{BG} 2) / 2$, prior to treatment was $19 \cdot 35(7 \cdot 5)$ photon counts/ms. This increased by a mean of 0.69 photon count $/ \mathrm{ms}$ to $20.04(7.6)$ photon counts $/ \mathrm{ms}$ at 60 minutes $(p=0 \cdot 38)$. Over the same time period the signal in the untreated eye also rose by a similar amount $(0.67$ photon count $/ \mathrm{ms}$ ) from $23.69(7 \cdot 4)$ photons $/ \mathrm{ms}$ at

Table 3 Pupillary dilatation and cèll count

\begin{tabular}{lll}
\hline \multirow{2}{*}{$\begin{array}{c}\text { Time } \\
(\min )\end{array}$} & Cell count: mean $(S D)$ & \\
\cline { 2 - 3 } & Untreated & Treated \\
\hline 0 & 0 & $0.02(0.06)$ \\
30 & $0.02(0.06)$ & $0.06(0.19)$ \\
60 & $0.04(0.08)$ & $0.14(0.38)$ \\
\hline
\end{tabular}


0 minutes to $24.36(7 \cdot 4)$ photons $/ \mathrm{ms}$ at 60 minutes $(p=0.44)$. There was no statistically significant difference between the change in mean background count and the change in signal over the 60 -minute period $(p=0.83)$. In the treated eye the mean background count prior to treatment was $18.0(7 \cdot 6)$ photon counts/ms, and this fell by a mean $0 \cdot 60$ photon count/ms to $17 \cdot 4$ $(7 \cdot 5)$ photons $/ \mathrm{ms}$ at 60 minutes $(p=0 \cdot 14)$. Over the same time period the mean signal fell by $0 \cdot 11$ photon $/ \mathrm{ms}(p=0.06)$. This decrease in the signal was statistically significantly greater than the fall in mean background count $(\mathrm{p}=0.03)$.

There was no significant difference in the cell count between the right and left eye prior to dilatation. However, the mean cell count recorded in the dilated left eye was greater than in the untreated right eye at both 30 and 60 minutes after instillation of the mydriatic. This increase did not reach statistical significance $(\mathrm{p}>0.05)$. In the majority of subjects $(8 / 10)$ no cells were detected either before or after dilatation. However, in one Asian subject with a brown iris the cell count increased from $0.2(0.4)$ before dilatation to $1 \cdot 2(0 \cdot 8)$ at 60 minutes.

\section{Discussion}

The Kowa laser flare cell meter is a sophisticated instrument that can quantify protein concentration and cells in the anterior chamber and has great clinical potential. In the assessment of any new instrument an estimate of its reproducibility and reliability is essential before further data analysis can be performed.

Using our calibration curve and the linear regression formula obtained, we found the mean anterior chamber protein concentration to be 111 $\mathrm{mg} / \mathrm{l}$. Sawa et $a l^{1}$ in their study of the laser flare cell meter and using bovine serum albumin found a mean concentration of $270 \mathrm{mg} / \mathrm{l}$ and Yoshimoto et $a l^{4}$ also using bovine serum albumin found a concentration of 157-186 $\mathrm{mg} / \mathrm{l}$. All these values are consistent with previous laboratory findings with aqueous samples obtained by paracentesis. ${ }^{5}$ The discrepancy between our results and those of Sawa et $a l^{1}$ and Yoshimoto $e t a l^{4}$ may be explained by the different type and purity of albumin used in their studies.

In our study the coefficient of variation of protein-related photon/ms counts repeated after an interval of 5 minutes was $8 \cdot 26 \%$. Oshika $e t a l^{2}$ using a similar experimental method found a coefficient of reproducibility of $12.5 \%$. Our results, when calculated in an identical manner to that of Oshika $e t a l,{ }^{2}$ give a value of $9 \cdot 54 \%$, which compares favourably with the reported reproducibility of anterior segment fluorophotometry. ${ }^{6}$ These data, together with the calculation of the smallest difference between two groups detectable by the instrument, would indicate that the Kowa FC1000 is sufficiently accurate and reliable to allow an analysis of the effect of various factors on photon count/ms values derived from aqueous protein concentrations.

Oshika $e t a l$ have shown a diurnal variation in aqueous flare over a 24-hour period with a peak at 0600 and a trough at 1800 . They found that there was a statistically significant increase in photon counts $/ \mathrm{ms}$ at 0200,0600 , and 1000 when compared with the value at 1800 the previous day. Further analysis of their data between 1000 and 1800 on the same day would be of interest. Although there was a decrease of 0.51 photon count $/ \mathrm{ms}$ over this period, it is not possible from their data to ascertain whether this change is in fact statistically significant over the same working day.

In our study all measurements were performed between 1000 and 1900. Multiple regression analysis of all variables, including time of day, demonstrated that there was no significant variation in photon counts/ms during this 9-hour period. This finding may be due to the small sample size but could be of great practical importance, as it implies that the circadian effect during the working day on measurements in normal eyes is small, and measurements do not necessarily have to be performed at the same time of the working day.

Oshika $e t a l$ also found that there was no significant difference between measurements performed at the same time of day at an interval of 24 hours. In our study we assessed subject variability at an interval of one week, with measurements being performed at the same time of day. There was no significant difference between these two measurements, and the average coefficient of variation between these measurements was $12 \cdot 2 \%$. After allowing for the coefficient of reproducibility and diurnal variation this would indicate that there is little subject variability in photon counts/ms and hence aqueous protein concentration over this period of time.

In our study we found no significant difference in photon counts/ms between right and left eyes. A similar finding was reported by Sawa et al. ${ }^{1}$ In-vitro measurements on aspirated aqueous humour have previously shown a difference in protein concentrations between right and left eyes, ${ }^{7}$ but our study would indicate that any such difference does not have any significant effect on the photon count/ms.

We have found a significant correlation between age and photon counts/ms. Sawa et al compared photon counts/ms in a group of young adults and elderly patients with early cataract and found significantly higher counts in the elderly age groups. Oshika $e t a l^{8}$ have found a similar increase in photon counts/ms with age. Several factors may explain this increase in photon counts/ms. It may represent a true increase in the total protein concentration in the aqueous, or a change in aqueous composition. Fluorophotometry has demonstrated a breakdown of the blood-aqueous barrier with age, ${ }^{6}$ and in-vitro electrophoretic studies have shown an increase in total protein concentration ${ }^{9}$ and also immunoglobulin concentrations with age. ${ }^{5}$ Inada $e t a l^{10}$. using crossed immunoelectrophoresis of aqueous humour, have also found an increase in the concentration of prealbumin, albumin, $\alpha-1$ acid glycoprotein, and transferrin with age.

Another factor contributing to an increase in photon counts/ms with age may be light scatter from within the lens or reflection from the lens surface, which would be expected to be greater in cataractous eyes. In our study this is unlikely, as 
subjects of all ages with visual acuities of $6 / 6$ or better and clinically clear lenses were chosen. Despite this we still found significantly higher photon counts/ms in all age groups above 40 compared with values in the under 30 age group.

Sawa $e t a l^{l}$ investigated the effect of pupillary dilatation and found that the photon count/ms decreased with dilatation and obtained a ratio of 0.91 (SD 0.25 ) between the dilated and undilated eyes of 31 normal adults, which was not statistically significant. However, Oshika and $\mathrm{Kato}^{11}$ in a more comprehensive study of eyes found a significant decrease in photon counts/ms at $3 \mathrm{~h}$ following instillation of $5 \%$ phenylephrine and by $1 \mathrm{~h}$ following instillation of both $0.5 \%$ phenylephrine - tropicamide $0.5 \%$ and tropicamide $0.4 \%$ alone. They attributed this change to a pharmacological effect of the mydriatic.

In our study we found a ratio of $0 \cdot 89$ (SD $0 \cdot 10$ ) at 30 minutes after instillation of tropicamide and a ratio of 0.85 (SD 0.09) at 60 minutes, the latter representing a statistically significant change from the predilatation ratio of 0.98 (SD $0.12)(p=0.002)$. Whether this decrease in photon count/ms represents either a true change in aqueous composition or an artefactual change due to a change in background scatter from a dilated pupil is unknown, but dilatation does present a more homogeneous background to the measurement site, and thus minimises the effect of light scattering by surrounding ocular tissues such as the iris. Our analysis of mean background counts suggests that, although there is a reduction in background light scatter, with mydriasis this does not completely explain the decrease in photon counts/ms observed. Other possible explanations for a true change in the aqueous protein concentration or composition could be either a pharmacologically induced change in aqueous flow rate or a change in the permeability of blood vessels in the anterior uvea. Although there have been studies on the effect of phenylephrine on these parameters, ${ }^{12}{ }^{13}$ we are not aware of any comparable studies on tropicamide with human eyes.

In conclusion, our results indicate that the Kowa $\mathrm{FCl} 1000$ is an accurate and sensitive instrument with potential applications in the clinical investigation of damage to the bloodaqueous barrier.

This study was supported by charitable funds from the Star Foundation and the Andrew Wilson Trust through Dr Elizabeth Frankland Moore.

The statistical advice and help given by Mr Nick Taub is The statistical advice and help given by Mr Nick Taub is
ratefully acknowledged. We thank Mrs J M Andrews for her secretarial services.

1 Sawa $M$, Tsurimaki Y, Tsuru H, Shimuzu M. New quantitative method to determine protein concentration and cell number in aqueous in vivo. $f \mathrm{pn} f$ Ophthalmol $1988 ; 32: 132-$ 42.

2 Oshika T, Araie M, Masuda K. Diurnal variation of aqueous flare in normal human eyes measured with laser flare cell meter. F pn 7 Ophthalmol 1988; 32: 143-50.

3 Everitt BS. Statistical models for medical investigations. Oxford: Oxford University Press, 1989.

4 Yoshimoto T, Wong AS, Daher E, Sears ML. Aqueous flare measurement with laser flare-cell meter. $\mathcal{F p n} \mathcal{F}$ Ophthalmol $1990 ; 34: 57-62$.

5 Zirm M. Proteins in aqueous humor. Adv Ophthalmol 1980; 40: $100-72$.

6 Fearnley I, Spalton DJ, Smith SE. Anterior segment fluorophotometry in acute anterior uveitis. Arch Ophthalmol 1987; 105: 1550-5.

7 Kronfeld PC. The protein content of the aqueous humor in man. Am f Ophthalmol 1941; 24: 1121-31.

8 Oshika T, Kato S, Sawa M, Masuda K. Aqueous flare intensity and age. $\mathcal{F p n} \mathcal{F}$ Ophthalmol 1989; 33: 237-42.

9 Krause U, Raunio V. The protein of the pathologic human aqueous humor. Ophthalmologica $1970 ; 16: 280-7$.

10 Inada K, Murata T, Baba H, Murata Y, Ozaki M. Increase of aqueous humor proteins with ageing. $\mathcal{f} p n \mathcal{f}$ Ophthalmol 1988; 32: 126-31.

11 Oshika T, Kato S. Changes in aqueous flare and cells after mydriasis. Fpn f Ophthalmol 1989; 33: 271-8.

12 Genderen MM, Best JA, Oosterhuis JA. The immediate effect of phenylephrine on aqueous flow in man. Invest Ophthalmol Vis $S_{c i}$ 1988; 29: 1469-73.

13 Araie M. Acute effects of topical phenylephrine on aqueous humor dynamics and corneal endothelial permeability in man. Fpn f Ophthalmol 1983; 27: 340-5. 